\title{
Gastric Mucosal Cell Proliferation in Ethanol-Induced Chronic Mucosal Injury Is Related to Oxidative Stress and Lipid Peroxidation in Rats
}

\author{
Rolando Hernández-Muñoz, Coral Montiel-Ruíz, and Olivia Vázquez-Martínez \\ Departamento de Biología Celular, Instituto de Fisiología Celular, Universidad Nacional Autónoma de México \\ (UNAM), Mexico
}

\begin{abstract}
SUMMARY: The oxygen free radicals-induced lipid peroxidation (LP) has been implicated in the pathogenesis of acute ethanol-induced gastric mucosal lesions. However, the role of LP in the generation of chronic gastric mucosal injury is unknown. We have developed a model of chronic mucosal injury induced by continuous ethanol ingestion for 5 days and characterized by marked alterations in plasma membranes from gastric mucosa. Therefore, LP was evaluated in this experimental model. Indicators of peroxidative activity, mucosal glutathione content, thymidine kinase activity (an index of cell proliferation), and histamine $\mathrm{H}_{2}$-receptor $\left(\mathrm{H}_{2} \mathrm{R}\right)$ binding constants were quantified in animals undergoing gastric mucosal damage. The effect of famotidine, $\mathrm{a} \mathrm{H}_{2} \mathrm{R}$ antagonist that readily ameliorates the chronic mucosal injury, was also tested. Increased free radicals and LP levels were detected during gastritis; however, a second, higher peak of LP was noted in mucosal plasma membranes after ethanol withdrawal (recovery period). This further increase of LP coincided with active cell proliferation, decreased mucosal glutathione levels, and diminished specific cimetidine binding by $\mathrm{H}_{2} \mathrm{R}$. Administration of famotidine accelerated the mucosal proliferative process, inducing the second lipoperoxidative episode sooner, and preserved the content of glutathione. In addition, LP correlated directly with cell proliferation and inversely with mucosal membrane cimetidine binding. In conclusion, LP seems to be involved in chronic ethanol-induced gastric mucosal injury. However, a further enhancement of plasma membrane LP occurred, associated with increased DNA synthesis and diminished cimetidine binding by membrane $\mathrm{H}_{2} \mathrm{R}$. Therefore, increased LP could also participate in the compensatory mucosal proliferation initiated after ethanol withdrawal. (Lab Invest 2000, 80:1161-1169).
\end{abstract}

$$
T
$$
here is growing evidence that lipid peroxidation (LP) could play a significant role in the pathogenesis of ethanol-induced gastric mucosal lesions, especially because oxygen radicals have been directly implicated in the damage of cell membranes after administration of that agent (Kvietys et al, 1990; Szelenyi and Brune, 1988; Terano et al, 1989). Indeed, the hypothesis that superoxide free radicals are involved in the immediate deleterious effect of ethanol on gastric mucosa seems to be confirmed by these studies. However, there are no reports regarding the involvement of LP in a model of mucosal damage induced by the long-term administration of ethanol.

We have found that ethanol administration elicits a histological profile of chronic gastric injury, accompanied by evident alterations in the plasma membranes obtained from gastric mucosa (Hernández-Muñoz and Montiel-Ruíz, 1996). These alterations (ie, increased cholesterol-phospholipids ratio, lower membrane fluidity, and diminished activities of some gastric mucosa membrane-associated enzymes), might be at-

Received January 21, 2000.

Address reprint requests to: Dr. Rolando Hernández-Muñoz, Departamento de Biología Celular, Instituto de Fisiología Celular, Universidad Nacional Autónoma de México (UNAM), Apartado Postal 70-243, México 04510, D.F., Mexico. Fax: 525-622-5611; E-mail: rhernand@ifcsun1.ifisiol.unam.mx tributed to an enhanced rate of LP (Hernández-Muñoz and Montiel-Ruíz, 1996).

In the present model of ethanol-induced mucosal injury, a gradual restoration of the surface epithelium of gastric mucosa occurred after ethanol withdrawal and was readily accelerated by the treatment with antagonists of the histamine $\mathrm{H}_{2}$-receptor $\left(\mathrm{H}_{2} \mathrm{RA}\right)$. We suggested previously that the beneficial effect of the $\mathrm{H}_{2} \mathrm{RA}$ on ethanol-induced mucosal injury might be related to increased proliferative events promoted by $\mathrm{H}_{2} \mathrm{RA}$ during the reversal of gastric mucosal damage (Hernández-Muñoz and Montiel-Ruíz, 1996). This putative action of $\mathrm{H}_{2} \mathrm{RA}$ could be through modulation of histamine $\mathrm{H}_{2}$ receptors $\left(\mathrm{H}_{2} \mathrm{R}\right)$, because the number and affinity of these receptors change during proliferation and differentiation of normal epithelial and gastric carcinoma cells (Arima et al, 1991).

Therefore, the present study was aimed at evaluating the involvement of oxidative stress and LP during the onset of ethanol-induced gastric mucosal injury and its subsequent reversal. Data strongly suggest that LP participates in the pathogenesis of ethanolinduced subchronic gastritis. However, a further enhancement of plasma membrane LP occurred associated with increased cell proliferation in the damaged gastric mucosa and quite similar to that found in another model of cellular proliferation, namely that 
which occurs during liver regeneration (Aguilar-Delfín et al, 1996).

\section{Results}

\section{Oxidative Reactions Associated with the Onset of and Recovery from Subchronic Gastritis}

The levels of thiobarbituric acid-reactive substances (TBARS), conjugated dienes, and luminol-dependent chemiluminescence, as parameters used to ensure an estimation of peroxidative events, were measured in subcellular fractions isolated from gastric mucosa. Determinations were performed with animals subjected to chronic mucosal injury and those receiving the $\mathrm{H}_{2} \mathrm{RA}$.

The control plasma membrane level of TBARS (1.6 $\pm 0.2 \mathrm{nmol} / \mathrm{mg}$ of protein) was significantly changed by ethanol administered by gavage 6 hours earlier $(2.5 \pm 0.2 \mathrm{nmol} / \mathrm{mg} ; p<0.05)$. At the onset of gastric mucosal damage $(\mathrm{h}=0)$ (Fig. 1A), TBARS content increased more than 3-fold compared with control mucosa. After ethanol withdrawal, the level of TBARS rapidly declined, normalizing at 48 hours, and then suddenly increased in this subcellular fraction (72 hours). This second peak of plasma membrane TBARS in the gastric mucosa was even higher than that found at onset (8- to 9-fold over controls) (Fig. 1A). Thereafter, the amount of TBARS was promptly normalized, and 7 days after ethanol withdrawal, it returned to basal values $(1.8 \pm 0.3 \mathrm{nmol} / \mathrm{mg}$ of protein). The administration of famotidine to rats subjected to gastric mucosal injury significantly changed the profile of plasma membrane TBARS levels. The $\mathrm{H}_{2} \mathrm{RA}$ induced an earlier peak of TBARS in the plasma membranes of animals with gastric mucosal injury. TBARS peaked at 48 hours after treatment (6- to 8-fold over controls) and returned to normal levels at 72 hours (Fig. 1A).

The amount of TBARS in the cytosolic fraction (control value: $0.6 \pm 0.2 \mathrm{nmol} / \mathrm{mg}$ of protein) was slightly modified at the onset of chronic mucosal damage (not shown). However, cytosolic TBARS were significantly enhanced at 24 hours $(1.1 \pm 0.2 \mathrm{nmol} /$ mg; $p<0.05)$, normalized at 48 hours, and again elevated at 72 hours after ethanol withdrawal (1.2 \pm $0.2 \mathrm{nmol} / \mathrm{mg}$ ), which closely resembles the pattern that occurs in plasma membranes. Administration of famotidine only elicited minor changes in the damaged gastric mucosa-induced pattern of LP (TBARS content) in this subcellular fraction (data not shown). Microsomal production of TBARS $(0.7 \pm 0.1 \mathrm{nmol} / \mathrm{mg}$ of protein, in controls) was not modified by chronic mucosal injury (data not shown).

Control animals, which were not treated with ethanol but received the same schedule of famotidine administration as did the test animals, did not significantly show altered LP profiles, as assessed by TBARS quantification, in the three subcellular fractions tested (data not shown).

The insaturation rearrangement forming conjugated dienes (CD) indicates the magnitude of the propagative phase of the free radicals-induced LP (HernándezMuñoz et al, 1997). Figure 1 also suggests the effects of gastric mucosal injury and treatment with famotidine on this parameter. The amount of CD found in this subcellular fraction closely resembled that found in the rate of TBARS production, but to a lesser magnitude. At the onset of chronic mucosal damage, there were

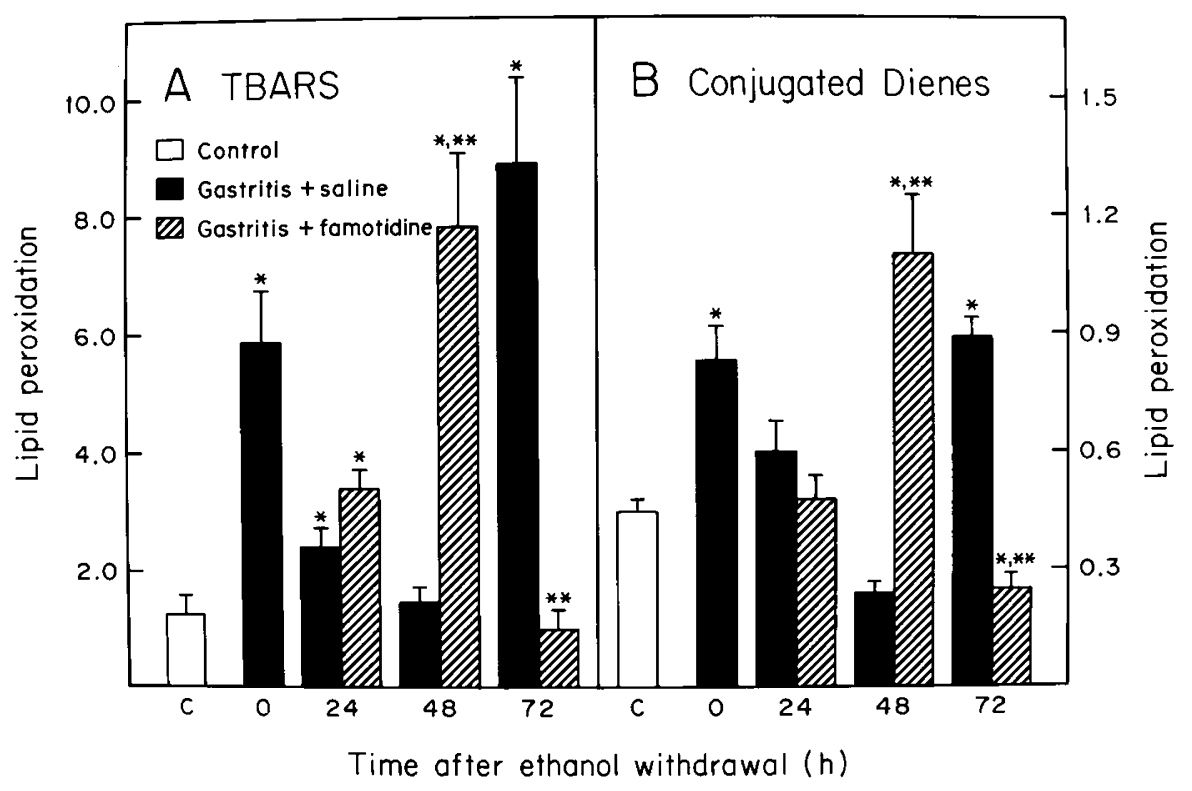

Figure 1.

Rate of lipid peroxidation (LP) in plasma membranes from rats subjected to ethanol-induced gastric mucosal injury and treated with famotidine. The rate of LP was estimated by the level of thiobarbituric acid-reactive substances (TBARS) production (A) and the amount of conjugated dienes (CD) (B) in plasma membranes isolated from gastric mucosa. TBARS are expressed as nmoles and CD as absorbance at $233 \mathrm{~nm}$ per milligram of protein. Symbols for each group are represented at the top of the figure (animals with mucosal damage are called "gastritis"). Results are the mean \pm SE of at least seven animals per group. Statistical significance: * $p<$ 0.01 vs controls; ${ }^{\star *} p<0.01$ vs gastritis subjects. 
higher levels of CD compared to the control group, but they were rapidly normalized (24 hours), decreased after 48 hours, and reached a maximal peak at 72 hours after ethanol withdrawal (Fig. 1B). Treatment with famotidine shifted the profile of CD in plasma membranes from rats undergoing mucosal damage. The $\mathrm{H}_{2} \mathrm{RA}$ inverted the CD pattern, inducing a drastic reduction in this parameter within 72 hours after ethanol withdrawal (Fig. 1B).

The luminol-dependent chemiluminescence (CL) represents the occurrence of short-lived free radicals and is, therefore, a sensitive assay for oxidative radical reactions (Hernández-Muñoz et al, 1997). This indicator of oxidative damage was also measured in the membrane and cytosolic fractions obtained from gastric mucosa. Plasma membrane $\mathrm{CL}$ was practically unaffected in the damaged mucosa or by treatment with famotidine, except for a significant decrease of $\mathrm{CL}$ in subcellular fractions obtained from animals with mucosal injury and receiving $\mathrm{H}_{2} \mathrm{RA}, 48$ hours after ethanol withdrawal (Table 1). The cytosolic rate of $\mathrm{CL}$ in animals subjected to gastritis was lower than that found in controls, but it returned to basal values thereafter. While the cytosolic rate of $\mathrm{CL}$ remained constant in animals subjected to ethanol-induced chronic mucosal injury during the recovery period, famotidine promoted a significant decrease of this parameter at 48 hours after ethanol withdrawal (Table 1). We did not find any significant change in the cytosolic rate when it was tested in the microsomal fraction obtained from the experimental groups (not shown). Moreover, administration of famotidine to control animals had no effect in either CD levels or the rate of $\mathrm{CL}$ in the three subcellular fractions tested (not shown).

The increased LP found after ethanol withdrawal in the group with damaged gastric mucosa was accompanied by a striking diminution of mucosal glutathione content, which reached its lowest value at the onset of the second peak of membrane TBARS level (Table 1). In contrast, famotidine promoted a significant increase of glutathione (at $48-72 \mathrm{hr}$ ), when the rate of LP was normalized by the administration of $\mathrm{H}_{2} \mathrm{RA}$ to these animals (Table 1$)$.

\section{Capacity of Gastric Mucosal Cells for DNA Synthesis and Proliferation}

The mitotic index, found in mucosal specimens from the experimental groups, is depicted in Figure 2A. After ethanol withdrawal, animals with injured gastric mucosa showed a gradual increase in mitotic images, reaching the maximum at 72 hours. Treatment with famotidine readily elicited an earlier and higher cellular proliferation when administered to animals undergoing chronic mucosal injury (Fig. 2A). To roughly estimate the gastric proliferative competence in the damaged gastric mucosa, as well as its recovery and the effect of the $\mathrm{H}_{2} R A$, thymidine kinase (TK) activity was measured in the cytosolic fraction obtained from the experimental groups. After ending ethanol treatment $(\mathrm{h}=0)$, TK increased significantly (approximately 2.5-fold over control) (Fig. 2B). Within 24 hours after ethanol discontinuation, TK activity increased in animals undergoing mucosal injury (Fig. 2B). This was followed by an unexpected decrease by 48 hours and a maximal peak of activity at 72 hours after ethanol withdrawal (7-fold over control). The treatment with famotidine effectively modified TK activity in these animals after ethanol withdrawal. The $\mathrm{H}_{2}$ RA promoted an early and prolonged cell-proliferative response, as reflected by the high rate of the cytosolic TK activity found in gastric mucosa (9- to 12-fold over controls) (Fig. 2B). Animals with injured gastric mucosa, whether receiving or not receiving famotidine, showed basal levels of TK activity 7 days after ethanol withdrawal (data not shown). Moreover, the $\mathrm{H}_{2} \mathrm{RA}$ did not have a significant effect on TK activity by itself, when administered to control rats (data not shown). Therefore, the changes observed in the cytosolic TK activity closely correlated with the mitotic activity found in gastric mucosa specimens obtained from the experimental groups (Fig. 2).

Table 1. Effects of the Onset of Chronic Gastric Mucosal Injury and Treatment with Famotidine on the Levels of Total Glutathione and Chemiluminescence in Subcellular Fractions from Gastric Mucosa

\begin{tabular}{|c|c|c|c|}
\hline \multirow[b]{2}{*}{ Treatment } & \multicolumn{2}{|c|}{ Chemiluminescence } & \multirow[b]{2}{*}{ Total glutathione } \\
\hline & Plasma membranes & Cytosol & \\
\hline \multicolumn{4}{|l|}{ Gastritis and saline } \\
\hline Time zero & $1.05 \pm 0.17$ & $1.88 \pm 0.25^{\star}$ & $2.2 \pm 0.2^{\star}$ \\
\hline 24 hours & $0.67 \pm 0.11$ & $2.38 \pm 0.41$ & $2.3 \pm 0.3^{*}$ \\
\hline 48 hours & $0.95 \pm 0.15$ & $3.45 \pm 0.63$ & $2.5 \pm 0.2$ \\
\hline 72 hours & $1.01 \pm 0.14$ & $2.80 \pm 0.38$ & $1.9 \pm 0.3^{*}$ \\
\hline \multicolumn{4}{|c|}{ Gastritis and famotidine } \\
\hline 24 hours & $0.95 \pm 0.11$ & $2.94 \pm 0.17$ & $2.7 \pm 0.2$ \\
\hline 48 hours & $0.54 \pm 0.08^{* *}$ & $1.73 \pm 0.23^{*}, * *$ & $4.3 \pm 0.3^{*},{ }^{* *}$ \\
\hline 72 hours & $0.81 \pm 0.14$ & $2.28 \pm 0.17$ & $4.1 \pm 0.5^{\star},{ }^{* \star}$ \\
\hline
\end{tabular}

Results are the mean \pm SE for at least seven animals per group. The rate of chemiluminescence $(\mathrm{CL})$ is expressed as $\mathrm{cmp} \times 10^{6} / \mathrm{mg}$ of $\mathrm{protein}$, while glutathione values are expressed as $\mu \mathrm{mol} / \mathrm{g}$ of gastric mucosa. Treatments were applied after ethanol withdrawal (group with mucosal damage is called "gastritis").

Statistical significance: * $p<0.01$ vs control group; ${ }^{\star \star} p<0.01$ vs gastritis group. 


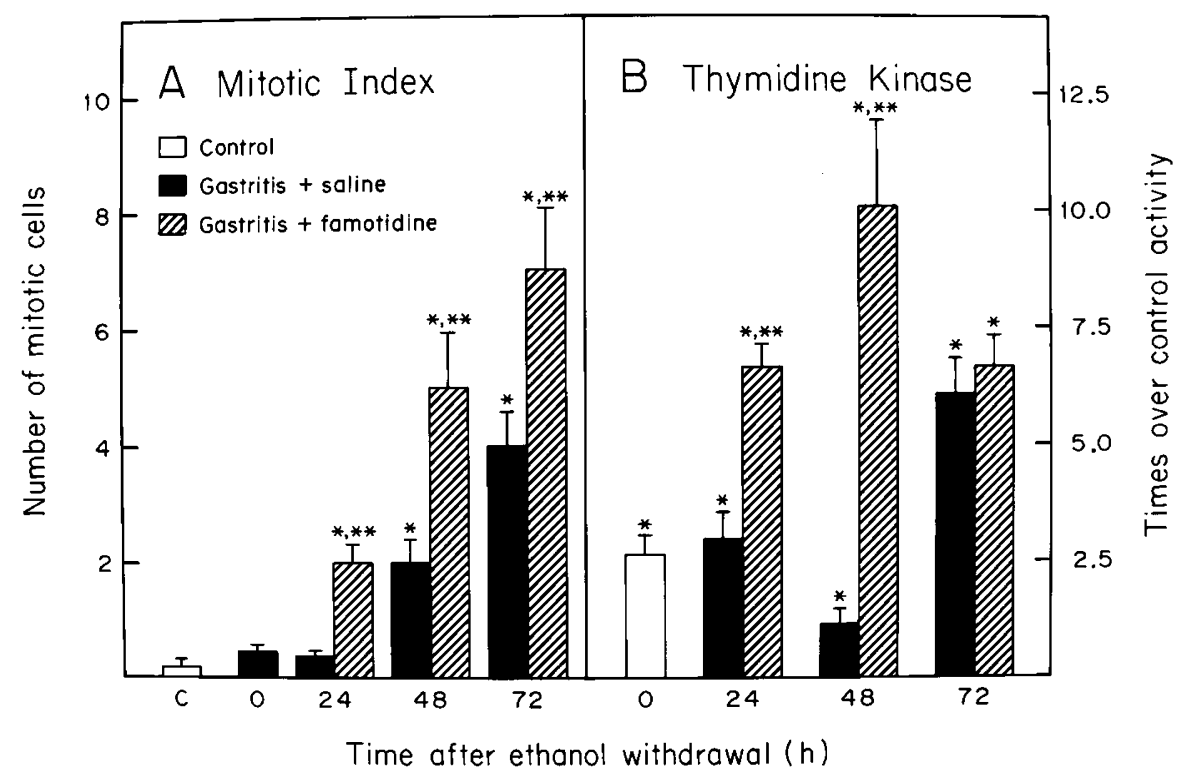

\section{Figure 2.}

Mitotic index and cytosolic thymidine kinase activity in gastric mucosa during mucosal injury induction, recovery period, and famotidine treatment. The mitotic index (mean $\pm \mathrm{SE}$ ) was microscopically assessed taking into account the number of mitotic cells in ten microscopic fields, with a $\times 40$ objective $(A)$. Thymidine kinase activity was measured in cytosol with at least six independent observations per experimental group, during the gastritis after ethanol withdrawal (B). Results are expressed as mean \pm SE of thymidine kinase activity $\left(0.21 \mathrm{nmol} / \mathrm{min} / \mathrm{mg}\right.$ of formed ${ }^{3} \mathrm{H}-\mathrm{TMP}$, in controls). Statistical significance is same as in Figure 1 .

\section{Modifications of Histamine $\mathrm{H}_{2}$-Receptors in Isolated Plasma Membranes from Gastric Mucosa}

Saturation isotherms of $\left[{ }^{3} \mathrm{H}\right]$ cimetidine binding were performed in highly purified plasma membranes to determine the presence of $\mathrm{H}_{2} \mathrm{R}$ in control gastric mucosa and the possible effect of LP on their density. The cimetidine binding was saturable and time- dependent, reaching equilibrium after 60 minutes (at $37^{\circ} \mathrm{C}$ ). Figure 3 shows the specific binding of $\left[{ }^{3} \mathrm{H}\right]$ cimetidine when the plasma membranes from normal gastric mucosa were incubated with the ligand in a range of 0 to $1 \mu \mathrm{M}$. The presence of two components was clear in the curve: the first corresponded to the cimetidine binding to plasma membranes at ligand

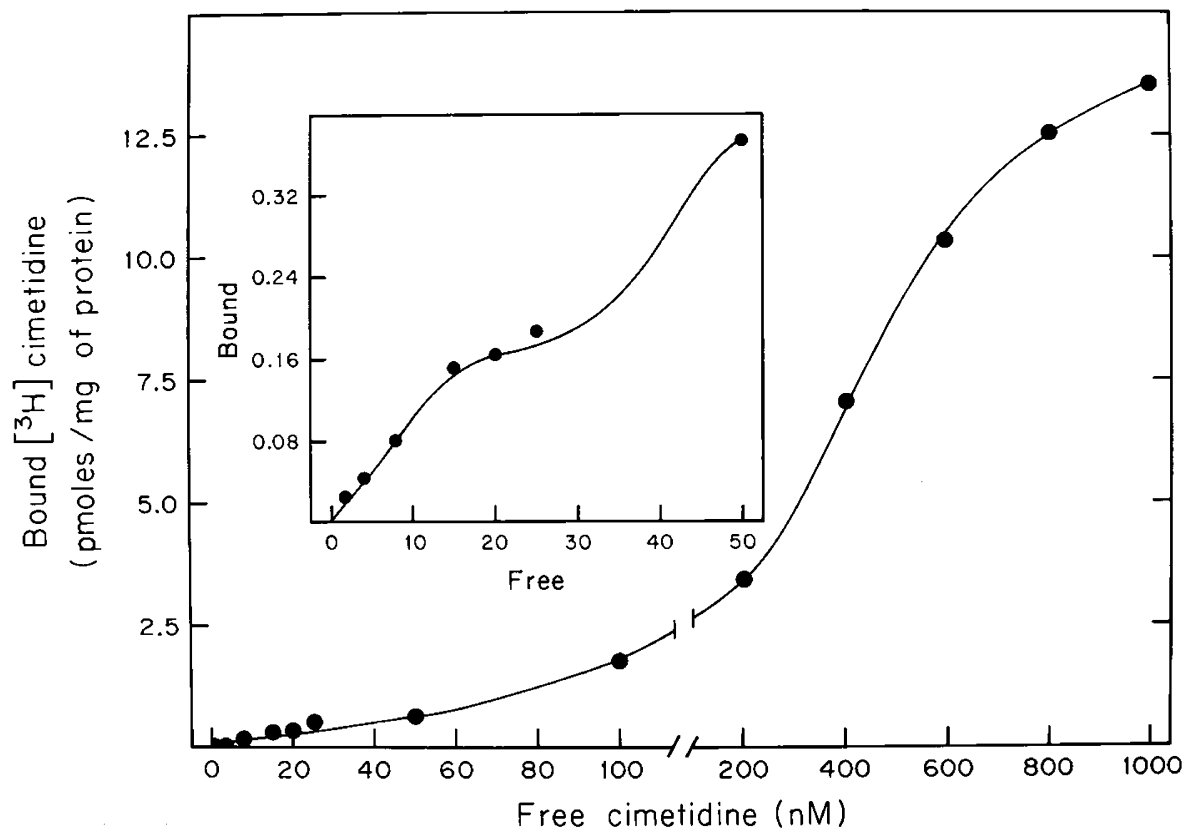

Figure 3.

Cimetidine receptor binding $\left(\mathrm{H}_{2}\right.$-histaminergic) in isolated plasma membranes of gastric mucosa obtained from control rats. The isotherm of [ ${ }^{3} \mathrm{H}$ cimetidine binding was obtained using 13 different concentrations for the ligand $(0-1 \mu \mathrm{m})$, and it is the average of five independent assays carried out three times each. Inset: Saturation isotherm in the lower range of cimetidine concentration $(2-50 \mathrm{~nm})$. 
concentrations below 40 to $50 \mathrm{~nm}$, and the second (not saturable at the concentrations tested) was performed at very high $\left[{ }^{3} \mathrm{H}\right]$ cimetidine concentrations. By Scatchard plot analysis, we found a high-affinity site for specific binding of cimetidine $\left(\mathrm{K}_{d}: 28 \mathrm{~nm}\right.$ for the ligand, and $B_{\max }$ of $270 \mathrm{fmol} / \mathrm{mg}$ of protein), whereas we failed to match the second site by Scatchard plot analysis (data not shown).

The $\left[{ }^{3} \mathrm{H}\right]$ cimetidine binding was also tested in plasma membranes obtained from the experimental groups, using $20 \mathrm{~nm}$ of cimetidine (below the $\mathrm{K}_{d}$ found for the high-affinity site for the $\mathrm{H}_{2} \mathrm{R}$ ). Figure 4 depicts the modifications of this binding induced by the onset of subchronic gastritis and its later recovery, as well as the effect of in vivo administration of famotidine. At the onset of chronic mucosal injury, the binding of cimetidine by isolated plasma membranes was drastically decreased. After ethanol withdrawal (24 and $48 \mathrm{hr}$ ), the binding of the antagonist was promptly restored, but it showed a second abrupt drop at 72 hours into the recovery period (Fig. 4). The treatment with famotidine of animals undergoing gastric mucosal lesion induced a different profile of cimetidine binding in mucosal plasma membranes. A single dose of famotidine (at 24 hr) did not affect the ligand binding in these animals; however, at 48 hours into the recovery period, a drastic inhibition of this parameter was noted. This was very transient, because, at 72 hours after ethanol withdrawal, $\left[{ }^{3} \mathrm{H}\right]$ cimetidine binding was practically the same as that in control animals (Fig. 4). Thereafter, the binding of cimetidine by plasma membranes was normalized in the experimental groups (4-7 days after ethanol withdrawal; not shown).
From these data, it seemed that high levels of LP coincided with increased cell proliferation, whereas LP appeared as a mirror image of the $\left[{ }^{3} \mathrm{H}\right]$ cimetidine binding. When we looked for a possible relationship among these parameters, we found a significant correlation between the levels of plasma membrane TBARS and the rate of cell proliferation, as assessed by measurement of TK activity $(r=0.87 ; p<0.001)$ (Fig. 5A). In addition, Figure 5B shows a very significant inverse correlation $(r=-0.95 ; p<0.001)$ between LP and the function of the $\mathrm{H}_{2} \mathrm{R}$, as estimated by $\left[{ }^{3} \mathrm{H}\right]$ cimetidine binding in purified plasma membranes from gastric mucosa. Therefore, the highly significant correlations found among these parameters suggest an association between LP and the proliferative response, which was evident after cessation of ethanol treatment (Fig. 5).

\section{Discussion}

The present study was designed to assess the possible LP involvement in alterations at the plasma membrane level of the gastric mucosa during the progress of an experimental chronic gastric mucosal injury induced by ethanol administration (Hernández-Muñoz and Montiel-Ruíz, 1996). In the present study, acute ethanol administration significantly modified enhanced TBARS production by gastric mucosa. These effects were also noticed during chronic mucosal damage, where parameters indicative of oxidant stress (TBARS and CD) were evidently increased and accompanied by an important decrease of mucosal glutathione. These findings strongly suggest that oxi-

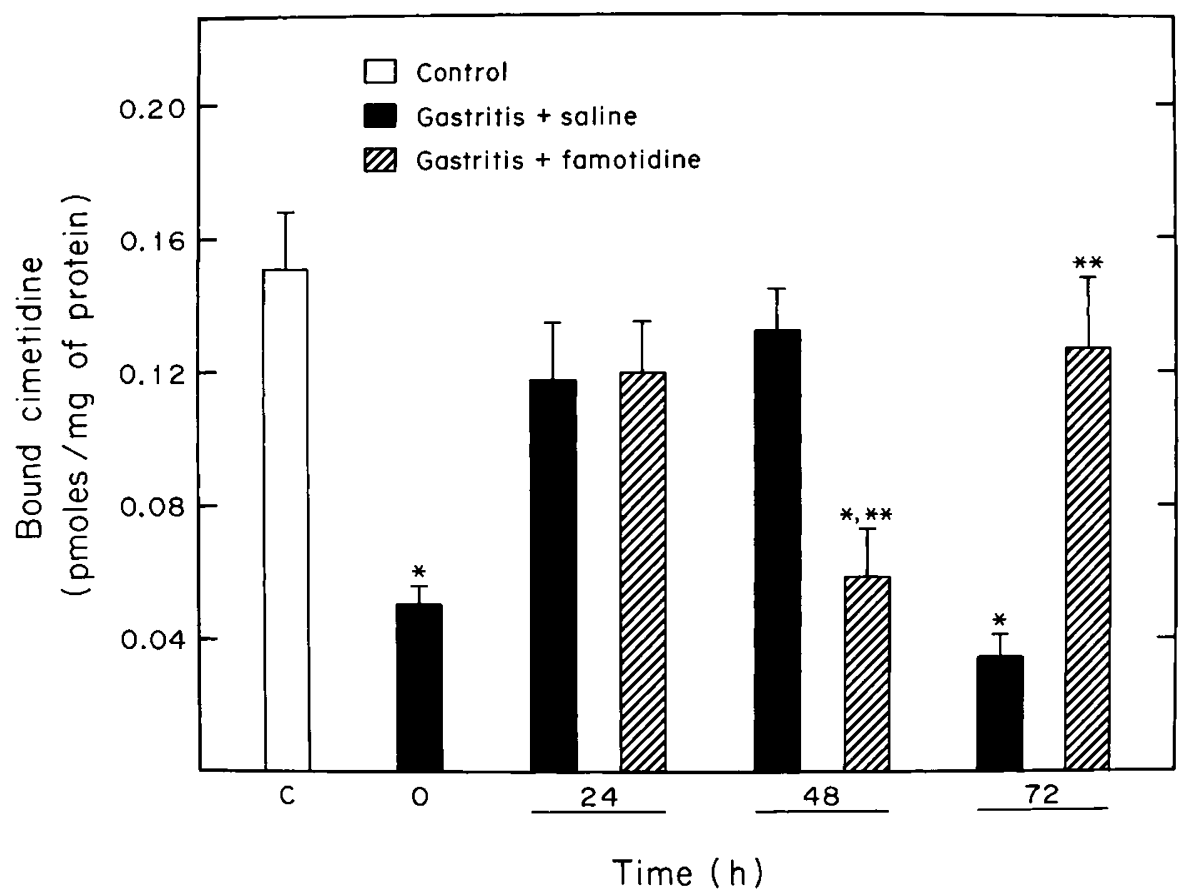

\section{Figure 4.}

$\left[{ }^{3} \mathrm{H}\right]$ Cimetidine binding in isolated plasma membranes from rats subjected to ethanol-induced mucosal damage and treated with famotidine. Results are the mean \pm SE of six independent observations by experimental group. The $\mathrm{H}_{2} \mathrm{RA}$ treatment was done after ethanol withdrawal (subchronic gastritis, $h=0$ ). Symbols per group and statistical analysis are as indicated in Figure 1. 


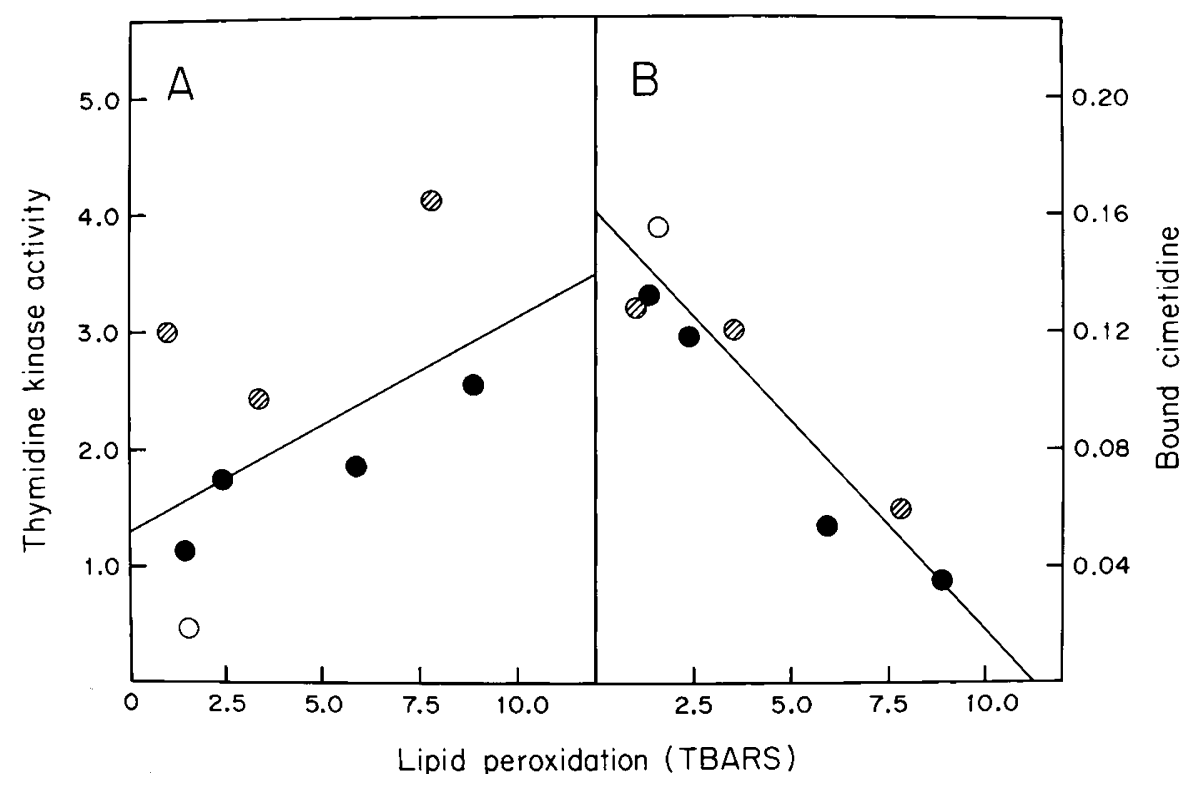

Figure 5 .

Correlation between LP and thymidine kinase (TK) activity and between LP and bound cimetidine in isolated plasma membranes from rats subjected to ethanol-induced gastric mucosal injury and treated with famotidine. Each point represents the average of six individual pairs of data. In A, data were taken from Figures 1 and 2 ; plot parameters: $r=0.87,(p<0.001)$, slope $=0.20$, and $\mathrm{y}$-intercept $=1.3 \mathrm{nmoles}$ of formed $\left[{ }^{3} \mathrm{H}\right] \mathrm{TMP} / \mathrm{min} / \mathrm{mg}$ of protein. Data for $\mathrm{LP}$ and $\left[{ }^{3} \mathrm{H}\right]$ cimetidine binding were taken from Figures 1 and $4 \mathrm{~B}$. Plot parameters: $r=-0.95$, $(p<0.001)$, slope $=-0.014$, and y-intercept $=0.16$ pmoles of bound cimetidine per milligram of protein. Symbols for each experimental group are as indicated in Figure 1.

dative stress took place during the generation of the gastric mucosa damage (as indicated by increased iluminol-dependent CL evoked by oxygen free radicals).

It has been recently suggested that LP plays a significant role in the generation of gastric mucosal lesions induced by several stresses (Yoshikawa et al, 1987). Indeed, oxygen radicals are capable of causing substantial gastric mucosal injury by a direct toxic effect, independent of luminal acid or mucosal blood supply (Esplugues and Whittle, 1989; Stein et al, 1989). The lipid hydroperoxides produced as a consequence would damage the biological membranes. Hence, mucosal damage can be easily produced by the generation of exogenous and endogenous active oxygen and free radicals (Yoshikawa et al, 1989).

Ethanol is involved in the formation of oxygen free radicals generated extra- and/or intracellularly. Because intragastric administration of superoxide dismutase was able to protect the gastric mucosa against the damaging effect of ethanol, this would suggest the involvement of superoxide free radicals in the pathogenesis of ethanol-induced gastric mucosal damage (Terano et al, 1989).

In addition, the depletion of glutathione enhances the LP initiated by superoxide radicals in both gastric mucosal homogenates and the gastric mucosa, as detected histochemically (Yoshikawa et al, 1997). In the present model of ethanol-induced chronic mucosal injury, decreased levels of mucosal glutathione persisted even during the recovery period (after discontinuation of ethanol). When measured at later periods after ethanol withdrawal (4-7 days), plasma membrane levels of TBARS and CD were found to be normalized, as were glutathione levels. However, the occurrence of an unexpected higher peak of LP was noted after 3 days of recovery (Fig. 1; Table 1).

The cause of this second major peak of LP-related products, occurring as it does after the recovery period in subcellular fractions of animals with gastritis, is more difficult to explain. Because this peak could be not related to oxidative stress, based upon the effect of famotidine, we were led to propose that LPmediated events might be associated with the restoration of the gastric mucosa after withdrawal from contact with the noxious agent.

An enhanced rate of plasma membrane LP, which was observed during the recovery period, could be attributed to a post-injury proliferative response of the gastric mucosa, in the same context that we have reported for the regenerating liver (Aguilar-Delfín et al, 1996). Indeed, LP promoted by partial hepatectomy is qualitatively distinct among subcellular fractions: it is transient and triggered only by an important loss of hepatic mass (above 40\%) and not accompanied by diminished levels of antioxidant metabolites, such as glutathione. Therefore, this event could be a normal cell event of physiological importance in the regenerating liver.

The enhanced rate of LP found in mucosal plasma membranes clearly coincided with the maximal activity of gastric mucosal thymidine kinase, a ratedetermining enzyme of DNA synthesis and considered to be a reliable marker of cell proliferation (Tsukamoto et al, 1996), and with the mitotic response against the injury. Animals treated with famotidine exhibited a high rate of peroxidative events (Fig. 1; Table 1) and of TK activity (Fig. 2). We selected the treatment with famotidine, among other $\mathrm{H}_{2} \mathrm{RA}$, based on its stronger 
beneficial effect on the ethanol-induced subchronic gastritis (Hernández-Muñoz and Montiel-Ruíz, 1996). Hence, these findings strongly suggest that increased LP found in the recovery period is closely linked to the proliferation of mucosal cells, a conclusion supported by the significant direct correlation between LP and TK activity (Fig. 5).

Regeneration of gastric surface cells occurs as a result of migration of epithelial cells from the gastric pits, suggesting that undamaged basal lamina is required for successful mucosal recovery (Black et al, 1985; Silen and Ito, 1985). This would suggest that beneficial agents not only provide protection to the gastric mucosa but may also allow mucosal reconstitution. Some antioxidants and scavenging agents for free radicals have been shown to prevent experimental short-term gastric mucosal damage (Yoshikawa et al, 1992). However, famotidine was able to accelerate reconstitution of gastric mucosa without exerting an antioxidant capacity in animals subjected to ethanolinduced subchronic gastritis. The effect of famotidine in stimulating the property of gastric mucosa cells to proliferate raised the question of whether there exists a possible involvement of $\mathrm{H}_{2} \mathrm{R}$ antagonism on mucosal cell regeneration.

It has been reported that cimetidine and ranitidine, but not famotidine, significantly delay and inhibit hepatic DNA synthesis and the percentage of mitosis in rats subjected to $70 \%$ partial hepatectomy (Kanashima et al, 1985; Kanashima and Kobayashi, 1989). Although the mechanism has not been clarified, $\mathrm{H}_{2} \mathrm{RA}$-induced changes in liver blood flow had no influence on their deleterious action on regenerating liver. Interestingly, both cimetidine and ranitidine had no effect on DNA synthesis or rate of mitosis when added to a hepatocyte culture at concentrations within the effective pharmacological dosage, and even 30 times higher, but serum from hepatectomized animals treated with these $\mathrm{H}_{2} \mathrm{RA}$ did inhibit in vitro proliferation of hepatocytes (Francavilla et al, 1989). These findings suggest that metabolite by-products of $\mathrm{H}_{2} \mathrm{RA}$ might affect the concentration of hormonal and growth factors in animals with regenerating liver.

The $\mathrm{H}_{2} \mathrm{RA}$ effects in modifying cell proliferation have also been found in gastric mucosal cells. Indeed, retinoic acid, which induces differentiation of normal epithelial and various carcinoma cells, increases histamine $\mathrm{H}_{2} \mathrm{R}$, without altering its affinity for tiotidine, in a human gastric carcinoma cell line (Arima et al, 1991). More recently, it has been shown that $\mathrm{H}_{2}$ blockers induce a dose-dependent stimulation of in vitro proliferation in the same adenocarcinoma cell line without significantly modifying cell migration (Ciacci et al, 1996). These authors have proposed that this mitogenic effect may contribute to the ulcer-healing effect of these $\mathrm{H}_{2}$-blockers in vivo. Although the differential effect of $\mathrm{H}_{2} \mathrm{RA}$ on the proliferative capacity in various tissues is unknown, we suggest that histamine $\mathrm{H}_{2} \mathrm{RA}$ could be deeply involved in the regulation of the cell cycle in cellular populations constituting the gastric mucosa.
The present kinetic analysis revealed the presence of a high-affinity site for specific binding of cimetidine $\left(\mathrm{K}_{d}: 28 \mathrm{~nm}\right.$ for the ligand and $\mathrm{B}_{\max }$ of $270 \mathrm{fmol} / \mathrm{mg}$ ) in highly purified plasma membranes from gastric mucosa. The assay showed a slightly higher affinity for the cimetidine than that reported in the gastric carcinoma cell line MKN-45 for $\left[{ }^{3} \mathrm{H}\right]$ tiotidine and a much higher affinity compared with the reported $\mathrm{K}_{d}$ of $5 \mu \mathrm{M}$ for the histamine binding site in gastric mucosal cells (Batzri et al, 1982).

Under our experimental conditions, we have demonstrated that lower cimetidine binding to plasma membranes is associated with increased rate of TBARS production in isolated plasma membranes from gastric mucosa. These findings suggest that antagonism of $\mathrm{H}_{2} \mathrm{R}$ could be somehow involved in the normal proliferative event which occurs after gastric damage induced by chronic administration of ethanol. The mechanism by which $\mathrm{H}_{2} \mathrm{RA}$ is capable of promoting cell proliferation in adenocarcinoma cell lines is unknown. However, it has been demonstrated that its action is not mediated by enhancing the effects of well-known proliferators of gastric mucosa, such as transforming growth factor-alpha (TGF- $\alpha$ ) or epidermal growth factor (EGF) (Ciacci et al, 1996). To our knowledge, the present data seem to be the first demonstration that treatment with $\mathrm{H}_{2} \mathrm{RA}$ can influence in vivo regeneration of gastric mucosal cells when given to animals subjected to ethanol-induced gastric mucosal damage.

In conclusion, LP induced by reactive oxygen radicals seemed to be involved in the generation of ethanol-induced chronic mucosal injury. However, regeneration of mucosal surface epithelium occurred after ethanol withdrawal and was accompanied by an important enhancement of LP in mucosal plasma membranes. This further peak of LP accompanied an increased DNA synthesis and a striking decrease in the capacity of membrane histamine $\mathrm{H}_{2}$-receptors to bind radioactive cimetidine. In addition, famotidine treatment of animals subjected to chronic mucosal damage accelerated cellular proliferation, leading to a histological and functional improvement of the gastric mucosa in rats (Hernández-Muñoz and Montiel-Ruíz, 1996). These findings revealed a significant correlation between LP and the magnitude of cell proliferation and an inverse correlation between LP events and the cimetidine binding by isolated plasma membranes from gastric mucosa.

\section{Materials and Methods}

\section{Materials}

$\left[\mathrm{N}-\right.$ methyl- $\left.{ }^{3} \mathrm{H}\right]$ cimetidine (specific activity $24 \mathrm{Ci} / \mathrm{mmol}$ ) and [Methyl- ${ }^{3} \mathrm{H}$ ] thymidine (specific activity $2 \mathrm{Ci} / \mathrm{mmol}$ ) were purchased from Amersham (Buckinghamshire, United Kingdom) and New England Nuclear Company (Boston, Massachusetts), respectively. Famotidine, Percoll, and other reagents were from SIGMA Chemical Company (St. Louis, Missouri). 


\section{Animal Treatments}

The present model of chronic ethanol-induced mucosal injury in male Wistar rats (230-270 g of body weight), has been recently reported in detail (Hernández-Muñoz and Montiel-Ruíz, 1996). Briefly, the animals, deprived of food overnight, received $1 \mathrm{ml}$ of $50 \%$ ethanol by intragastric gavage, followed by free access to food and $5 \%$ ethanol in water. The treatment was continued for five days, and on the fifth day ethanol was withdrawn (gastritis group, $\mathrm{h}=0$ ). The best reversion of ethanol-induced gastritis was accomplished by intragastric administration of $5 \mathrm{mg} /$ $\mathrm{kg} /$ day of famotidine for 1 to 3 days after ethanol withdrawal (Hernández-Muñoz and Montiel-Ruíz, 1996). Appropriate controls for each experimental condition were run simultaneously. All procedures were conducted in accordance with the Institutional Guide for Animal Experimentation (National University of Mexico).

\section{Subcellular Fractionation of Gastric Mucosa}

Under general anesthesia with sodium pentobarbital, stomachs were removed and rinsed in cold saline solution. Histological assessment of strips of gastric wall was performed as previously described (Hernández-Muñoz and Montiel-Ruíz, 1996). Rat gastric mucosa was totally excised and subcellular fractions were obtained as described in detail (HernándezMuñoz and Montiel-Ruíz, 1996). Plasma membranes with a high level of purity were obtained by the method of Loten and Redshaw-Loten (1986). Cytosolic and microsomal fractions were also obtained by differential centrifugation, and the purity of the fractions was assessed with marker enzyme activities (HernándezMuñoz and Montiel-Ruíz, 1996).

\section{Assays for Lipid Peroxidation and Free Radicals}

The amounts of some aldehydic products generated by LP were determined by the thiobarbituric acid reaction (Buege and Aust, 1978), modified as previously reported (Hernández-Muñoz et al, 1984), and the results listed as TBARS. Estimation of membrane LP-related CDs was performed as described by Recknagel and Glende (1984) and of free radicals, according to luminol CL (Cadenas and Sies, 1984). Gastric mucosa levels of glutathione (as an index of in vivo oxidative stress), were quantified in acid-extracts of gastric mucosa by a spectrophotometric method (Akerboom and Sies, 1981).

\section{Estimation of DNA Synthesis and Compensatory Cell Proliferation after Ethanol Withdrawal}

The cytosolic activity of thymidine kinase (EC 2.7.1.21), a reliable parameter for cell proliferation, was determined by the technique of Sauer and Wilmanns (1985). The mitotic index was quantified in histological samples stained with hematoxylin-eosin, and its magnitude expressed as described by Niemela et al (1995).

\section{$\left[{ }^{3} \mathrm{H}\right]$ Cimetidine Binding in Isolated Plasma Membranes of Gastric Mucosa}

To assess the specific binding of cimetidine by gastric mucosa, plasma membranes purified by Percoll gradients were harvested and washed three times in a buffer containing $250 \mathrm{mmol} / \mathrm{L}$ sucrose and $10 \mathrm{mmol} / \mathrm{L}$ TRIS-HCl, $\mathrm{pH}$ 7.4. The final pellet (100 $\mu \mathrm{g}$ of protein/ assay) was incubated in $500 \mu \mathrm{l}$ of the same buffer with increasing concentrations of $\left[\mathrm{N}\right.$-methyl $\left.{ }^{3} \mathrm{H}\right]$ cimetidine and the unlabeled ligand $(0.002-1 \mu \mathrm{M})$, in duplicate at $37^{\circ} \mathrm{C}$ for 60 minutes. After incubation, membranes were washed three times with sucrose-TRIS buffer and retained in Whatman GF/F filters. Dried filters were counted in $10 \mathrm{ml}$ of tritosol (Díaz-Muñoz et al, 1998). To determine the binding constants $\left(K_{d}\right.$ and $\mathrm{B}_{\text {max }}$ ), equilibrium experiments were subjected to Scatchard analysis. Nonspecific binding of $\left[{ }^{3} \mathrm{H}\right]$ cimetidine was defined by the addition of unlabeled 5 $\mathrm{mmol} / \mathrm{L}$ cimetidine or $1 \mathrm{mmol} / \mathrm{L}$ famotidine for each case. Hence, the specific binding was calculated by subtracting the nonspecific binding.

\section{Statistics}

All results are expressed as mean \pm SE The significance of the differences among groups was assessed by two-way ANOVA.

\section{Acknowledgements}

The authors thank Drs. J. Adolfo García-Sáinz, Rafael Villalobos-Molina, and Mauricio Díaz-Muñoz for their critical review of this manuscript, Ms. Susana Vidrio for her expert technical assistance, and Ms. María Elena Gutiérrez for her secretarial help.

\section{References}

Aguilar-Delfín I, López-Barrera F, and Hernández-Muñoz R (1996). Selective enhancement of lipid peroxidation in plasma membrane in two experimental models of liver regeneration: Partial hepatectomy and acute $\mathrm{CCl}_{4}$ administration. Hepatology 24:657-662.

Akerboom TPM and Sies H (1981). Determination of reduced and oxidized glutathione. Methods Enzymol 77:373-382.

Arima N, Yamashita Y, Nakata H, Nakamura A, Kinoshita Y, and Chiba T (1991). Presence of histamine $\mathrm{H}_{2}$-receptors on human gastric carcinoma cell line $\mathrm{MKN}-45$ and their increase by retinoic acid treatment. Biochem Biophys Res Commun 176:1027-1032.

Batzri S, Harmon JW, and Thompson WF (1982). Interaction of histamine with gastric mucosal cells: Effect of histamine $\mathrm{H}_{2}$-receptor antagonists on binding and biological response. Mol Pharmacol 22:41-47.

Black BA, Morris GP, and Wallace JL (1985). Effects of acid on the basal lamina of the rat stomach and duodenum. Virchows Arch B Cell Pathol Incl Mol Pathol 50:109-118.

Buege JA and Aust SD (1978). Microsomal lipid peroxidation. Methods Enzymol 52:302-310.

Cadenas E and Sies H (1984). Low-level chemiluminescence as an indicator of singlet molecular oxygen in biological systems. Methods Enzymol 105:221-231. 
Ciacci C, Zarilli R, Ricci V, De Luca A, Mazzaca G, DelVecchio-Blanco C, and Romano M (1996). Histamine $\mathrm{H}_{2}$ receptor antagonists stimulate proliferation but not migration of human gastric mucosal cells in vitro. Dig Dis Sci 41:972978.

Díaz-Muñoz M, Cañedo-Merino R, Gutiérrez-Salinas J, and Hernández-Muñoz R (1998). Modifications of intracellular calcium release channels and calcium mobilization following 70\% hepatectomy. Arch Biochem Biophys 349:105-112.

Esplugues JV and Whittle BJ (1989). Gastric damage following local intra-administration of reactive oxygen metabolites in the rat. Br J Pharmacol 97:1085-1192.

Francavilla A, Panella C, Polimeno L, Di Leo A, Makowka L, Barone M, Amoruso A, Ingrosso M, and Starzl TE (1989). Effect of cimetidine, ranitidine, famotidine, and omeprazole on hepatocyte proliferation in vitro. J Hepatol 8:32-41.

Hernández-Muñoz R, Díaz-Muñoz M, López V, LópezBarrera $F$, Yáñez L, Vidrio $S$, Aranda-Fraustro $A$, and Chagoya de Sánchez V (1997). Balance between oxidative damage and proliferative potential in an experimental rat model of $\mathrm{CCl}_{4}$-induced cirrhosis: Protective role of adenosine administration. Hepatology 26:1100-1110.

Hernández-Muñoz R, Glender W, Díaz-Muñoz M, GarcíaSáinz JA, and Chagoya de Sánchez V (1984). Effects of adenosine on liver cell damage induced by carbon tetrachloride. Biochem Pharmacol 33:2599-2604.

Hernández-Muñoz R and Montiel-Ruíz F (1996). Reversion by histamine $\mathrm{H} 2$-receptor antagonists of plasma membrane alterations in ethanol-induced gastritis. Dig Dis Sci 41:21562165.

Kanashima R and Kobayashi M (1989). Famotidine does not inhibit liver regeneration. Eur Surg Res 21:190-195.

Kanashima R, Nagasue N, and Sakato K (1985). Ranitidine as an inhibitor of liver regeneration. Am J Surg 149:223-227.

Kvietys PR, Twohig B, Danzell J, and Specian RD (1990). Ethanol-induced injury to the rat gastric mucosa. Role of neutrophils and xanthine oxidase-derived radicals. Gastroenterology 98:909-920.

Loten EG and Redshaw-Loten JC (1986). Preparation of rat liver plasma membranes in a high yield. Anal Biochem 154:183-185.

Niemela O, Parkkila S, Ylä-Herttuala S, Villanueva J, Ruebner B, and Halsted CH (1995). Sequential acetaldehyde production, lipid peroxidation, and fibrogenesis in micropig model of alcohol-induced liver disease. Hepatology 22:1208-1214.
Recknagel RO and Glende EA (1984). Spectrophotometric detection of lipid conjugated dienes. Methods Enzymol 105: 331-337.

Sauer H and Wilmanns W (1985). Thymidine kinase. In: Bergmeyer HU, editor. Methods of enzymatic analysis, vol. 3. Deerfield Beach: VCH Publishers, 468-473.

Silen W and Ito S (1985). Mechanisms for rapid reepithelialization of the gastric mucosal surface. Annu Rev Physiol 47:217-229.

Stein HJ, Esplugues JV, Whittle BJ, Bauerfeind P, Hinder RA, and Blum AL (1989). Direct cytotoxic effect of oxygen radicals on the gastric mucosa. Surgery 106:318-324.

Szelenyi I and Brune K (1988). Possible role of oxygen free radicals in ethanol-induced gastric mucosal damage in rats. Dig Dis Sci 33:865-871.

Terano A, Hiraishi H, Ota S, Shiga J, and Sugimoto T (1989). Role of superoxide and hydroxyl radicals in rat gastric mucosal injury induced by ethanol. Gastroenterol Jpn 24: 488-493.

Tsukamoto I, Yoshida Y, Kitamura Y, and Nomura S (1996). Inhibition of thymidylate synthase and thymidine kinase by okadaic acid in regenerating rat liver after partial hepatectomy. Biochem Pharmacol 52:879-884.

Yoshikawa T, Ichikawa H, Naito Y, and Kondo M (1992). Role of leukotrienes in gastric mucosal injury induced by ischemia-reperfusion in the rats. J Clin Gastroenterol 14: 568-570.

Yoshikawa T, Minamiyama Y, Ichikawa H, Takahashi S, Naito Y, and Kondo M (1997). Role of lipid peroxidation and antioxidants in gastric mucosal injury induced by the hypoxanthine- xanthine oxidase system in rats. Free Radic Biol Med 23:243-250.

Yoshikawa T, Ueda S, Naito Y, Takahashi S, Oyamada H, Morita Y, Yoneta T, and Kondo M (1989). Role of oxygenderived free radicals in gastric mucosal injury induced by ischemia or ischemia-reperfusion in rats. Free Radic Res Commun 7:285-291.

Yoshikawa T, Yoshida N, Miyagawa H, Takemura T, Tanigawa T, Sugino S, and Kondo M (1987). Role of lipid peroxidation in gastric mucosal lesions induced by burn shock in rats. J Clin Biochem Nutr 2:163-170. 